\title{
Normal Oropharynx. An Overlooked Obstruction
}

\author{
Liew Yew Toong*, Adzreil Bakri and Prepageran Narayanan \\ Department of Otorhinolaryngology, University Malaya Medical Centre, Kuala Lumpur, Malaysia
}

*Corresponding author: Liew Yew Toong, Department of Otorhinolaryngology, University Malaya Medical Centre, Kuala Lumpur, Malaysia

\section{Introduction}

A 35-year-old gentleman with underlying depression presented with noisy breathing and difficulty in breathing and was brought to emergency department by one of his family members. On examination, he was cyanosed and aphonia and an attempt of intubation was tried by an experienced anesthetist but failed. There was 'no airway' noticed except for pooling of secretions. The insertion of laryngeal mask airway also failed as there was resistance at the level of oropharynx. In view of rapid desaturation, cricothyroidotomy was performed to secure his airway. Computed tomography of the neck showed an expanded oropharynx (Figure 1). Examination under anesthesia revealed a huge plastic ball swallowed by the patient (Figure 2). It was removed and the cricothyroidotomy was converted to orotracheal intubation. Then he was successfully extubated the next day.

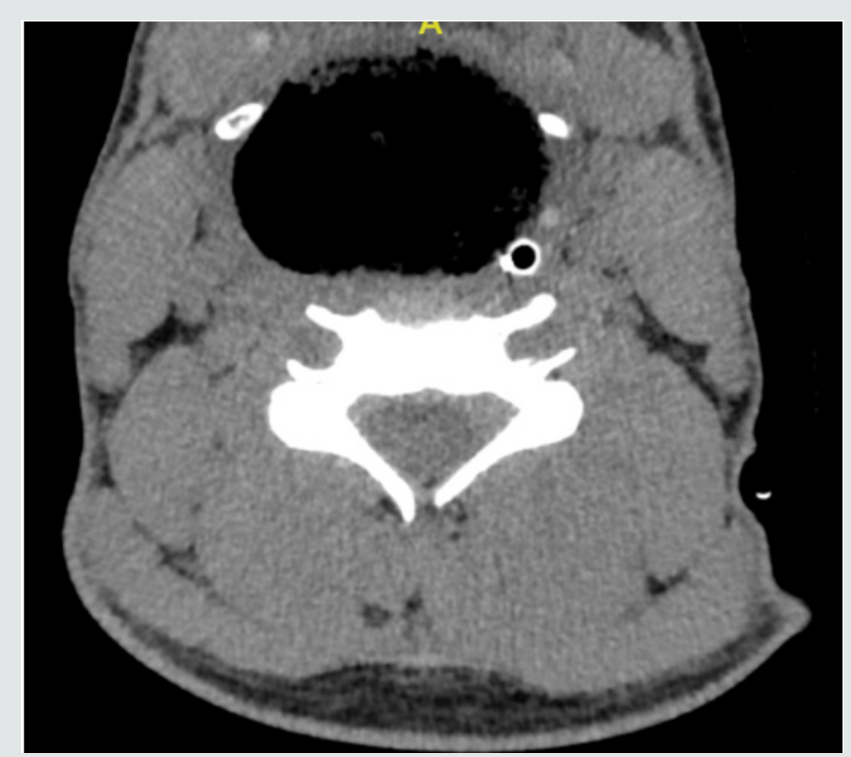

Figure 1.

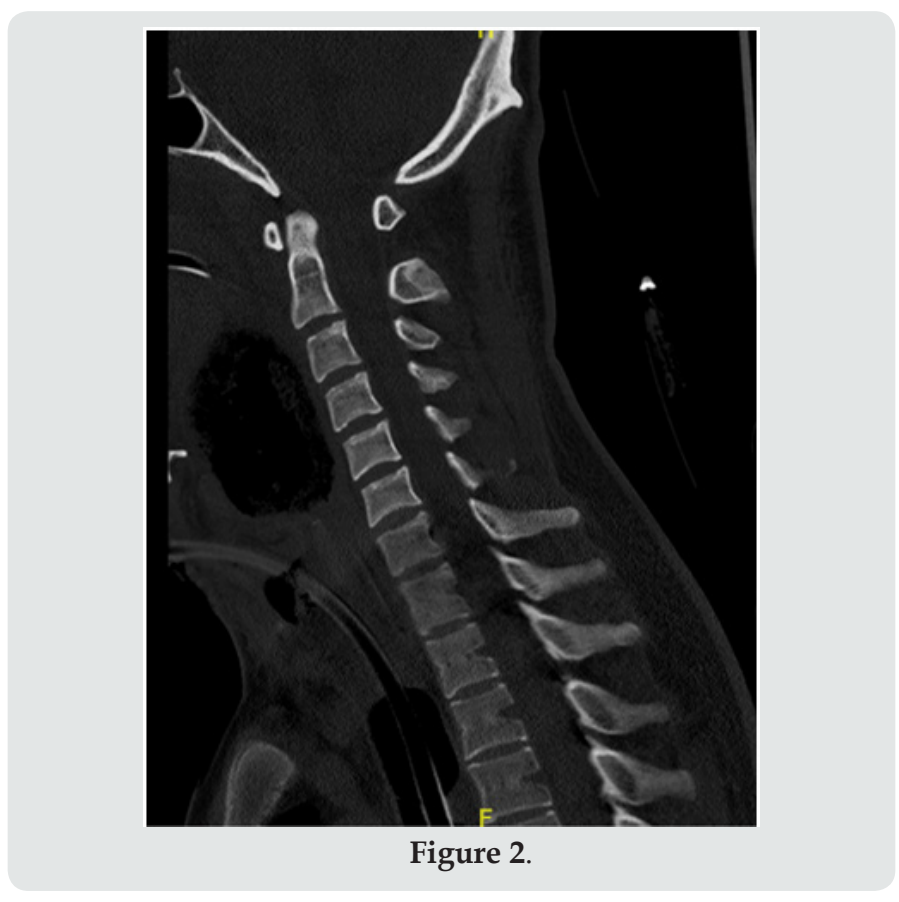

\section{Diagnosis}

Oropharyngeal translucent foreign body (plastic ball). Airway foreign body is uncommon in healthy young adults. There is a broad range of presentation from subtle to acute life-threatening airway obstruction. One large-city series of ambulance- transported airway foreign body patients reports a 3.3\% mortality (an average of one patient per month) in the prehospital phase alone [1]. History of ingestion of foreign body, inability to swallow and odynophagia are the most important diagnostic criteria [2]. However, it could be challenging among those mentally challenged patients where the history is usually unclear [3]. Therefore, emergency physicians must have high suspicion for foreign body impaction in mind 
in dealing with this group of patients who present with airway issues. Experienced anesthetist, otolaryngologist must be called in immediately to help out in this kind of difficult airway scenario. In our case, there was a totally occluded airway as the plastic toy ball stuck in oropharynx till cricothyroidotomy was needed. Imaging is usually a useful tool to help locate and delineate the foreign body, but it might be missed in unexperienced hands.

\section{Refernces}

1. Soroudi A, Shipp HE, Stepanski BM, Ray LU, Murrin PA, et al. (2007) Adult foreign body airway obstruction in the prehospital setting. Prehosp Emerg Care 11: 25-29.

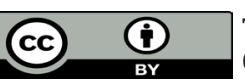

This work is licensed under Creative Commons Attribution 4.0 License

To Submit Your Article Click Here: Submit Article
2. Ong NP (1978) Foreign body in Oesophagus: Review of 2394 cases. Br J Surg 65: 5-9.

3. IS Sehgal, S Dhooria, B Ram, Singh N, Aggarwal AN, et al. (2015) Foreign body inhalation in the adult population: experience of 25,998 bronchoscopies and systematic review of the literature. Respiratory Care 60(10): 1438-1448.

DOI: $10.32474 /$ SJO.2019.02.000138

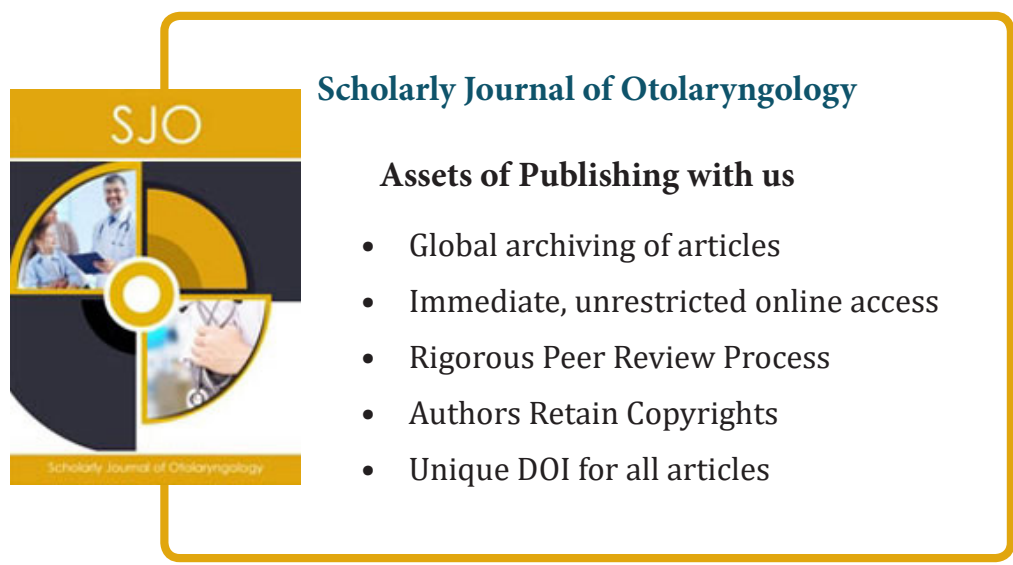

